\title{
Vertebrados terrestres del ecosistema Herbazal húmedo subnival del páramo en la Reserva de Producción de Fauna Chimborazo
}

\section{Terrestrial vertebrates of the ecosystem Subnival wet grassland of the paramo in the Chimborazo Fauna Production Reserve}

Sulaya Bayancela. ${ }^{1} \&$ Carlos Cajas. ${ }^{2}$

DOI: https://doi.org/10.33262/concienciadigital.v3i3.1285

\begin{abstract}
.
This paper presents an inventory of the diversity of terrestrial vertebrates in the subnival wet grassland paramo ecosystem (3400-4300 m.a.s.1.), of the Chimborazo Fauna Production Reserve (RPFCh), and analyzes the alpha and beta diversity indices of the wildlife. Field work was carried out between March and June 2019, in two sites of the ecosystem. The first was Pampas de Salasaca (3950 m.a.s.l. and $6^{\circ} \mathrm{C}$ temperature), located between two elevations on whose slopes, small patches of forest and little vegetation were observed, due to the abundant presence of cattle; mostly cloudy and rainy area. The second site was Siete Cochas $\left(3,860\right.$ m.a.s.l. and $\left.8^{\circ} \mathrm{C}\right)$, it presented a more compact structure with abundant vegetation, little presence of rain and no fog. A total of 17 species were registered, for birds ( 6 families, 4 orders and 10 species); and for land mammals ( 6 families, 5 orders, and 7 species). The results show that the specific richness for birds was 10 records (Margalef index $=2.36$, low medium diversity) and for mammals 7 (Margalef index $=1.48$, low medium diversity); for equity, birds (Shannon-Weaver Index $=1.90$, low mean diversity) and for mammals (Shannon-Weaver Index $=1.53$, low mean diversity); for dominance, birds (Simpson Index $=0.78$, low diversity) and mammals (Simpson Index $=0.72$, low diversity), concluding that this high-Andean ecosystem presented low diversity for birds and mammals.
\end{abstract}

\footnotetext{
${ }^{1}$ Escuela Superior Politécnica del Chimborazo, Facultad de Recursos Naturales, Chimborazo Ecuador, susasusa13@gmail.com

${ }^{2}$ Escuela Superior Politécnica del Chimborazo, Facultad de Recursos Naturales. Chimborazo Ecuador, carlos.cajas@espoch.edu.ec
} 
Keywords: inventory, diversity, vertebrates, terrestrial, ecosystem, grassland, subnival.

\section{Resumen.}

El presente artículo presenta el inventario de la diversidad de vertebrados terrestres en el ecosistema Herbazal húmedo subnival del páramo (3400-4300 m.s.n.m.), de la Reserva de Producción de Fauna Chimborazo (RPFCh), y analiza los índices de diversidad alfa y beta de la fauna. Entre marzo y junio de 2019 se realizó trabajo de campo en dos sitios del ecosistema. El primero, Pampas de Salasaca (3950 m.s.n.m. y $6^{\circ} \mathrm{C}$ de temperatura media), ubicado entre dos elevaciones en cuyas laderas se observó pequeños parches de bosque y una vegetación poco abundante por la presencia de ganado vacuno, zona mayormente nublada y con presencia de lluvia. El segundo sitio fue Siete Cochas (3860 m.s.n.m. y $8^{\circ} \mathrm{C}$ de temperatura media), el cual presentó una estructura más compacta con abundante vegetación, poca presencia de lluvia y ausencia de neblina. Se registró un total de 17 especies, para aves ( 6 familias, 4 órdenes y 10 especies); y para mamíferos terrestres (6 familias, 5 órdenes y 7 especies). Los resultados muestran que la riqueza específica para aves fue 10 registros (Índice de Margalef= 2,36, diversidad media baja) y para mamíferos 7 (Índice de Margalef = 1,48, diversidad media baja); para equidad, aves (Índice de Shannon-Weaver=1,90, diversidad media baja) y para mamíferos (Índice de Shannon-Weaver=1,53, diversidad media baja); para dominancia, aves (Índice de Simpson=0,78, diversidad baja) y mamíferos (Índice de Simpson=0,72, diversidad baja), concluyendo que este ecosistema alto-andino presentó diversidad baja para aves y mamíferos.

Palabras clave: inventario, diversidad, vertebrados, terrestres, ecosistema, herbazal, subnival.

\section{Introducción.}

Uno de los aspectos más sorprendentes de nuestro planeta es su biodiversidad. Según la agencia de las Naciones Unidas (PNUMA) en su Evaluación Global de la Biodiversidad se conoce 1.75 millones de especies, y estima que falta por describir entre 7 y 20 millones. Autores como (Mora et al. 2011) consideran que la diversidad biológica en el planeta está entre 1.2 y 1.3 millones de especies. Ecuador es un país megabiodiverso gracias a sus 91 ecosistemas (MAE, 2012), 4 regiones naturales, ubicación geográfica, cuencas hidrográficas, cordillera de los Andes, corrientes marinas y su historia geológica (Collot et al., 2009; Mena y Medina, 2001).

Estudios demuestran que países ubicados en la gradiente latitudinal 0 tienen mayor número de especies (Morales-Castilla y García-Valdés, 2014) debido al número constante de horas luz y estaciones anuales permanentes. Ecuador está entre uno de los 17 países en los cuales se concentra el 70\% de la biodiversidad del planeta (Bravo, 2014); registra 1.690 aves (Comité Ecuatoriano de Registros Ornitológicos, 2017), 440 especies de 
mamíferos (Tirira, 2018) y posee la mayor diversidad de especies de reptiles y anfibios del mundo, calculadas en 1033 entre ambas clases (Bioweb, 2020). Esta diversidad se concentra en los bosques húmedos tropicales, en los subtrópicos orientales y occidentales, y en los bosques alto andinos correspondientes al páramo (Lozano, 2017; Benítez et al. 2019). El páramo tiene una gradiente altitudinal que empieza desde los 3.000 m.s.n.m. hasta las nieves perpetuas (Hofstede et al., 2002). El páramo es un ecosistema vulnerable debido a la agricultura, ganadería, quemas, silvicultura y el cambio climático (PrietoTorres et al., 2015; León-García y Laso 2019; Farley et al. 2004; Beltrán et al. 2009); la fauna silvestre como Pseudalopex culpaeus, Puma concolor, Mustela frenata está amenazada por la introducción de especies exóticas como ratas, perros y gatos ferales que compiten por territorialidad y alimentos (Schipper et al., 2008; Traveset, \& Santamaría, 2004). Varios estudios reconocen que el páramo guarda diversidad biológica y endemismo (Balslev y Luetyn 1992; Luteyn 1999) sabiendo que las cadenas montañosas son barreras geográficas que limitan la distribución de las especies sobre todo de micromamíferos terrestres (Benítez et al. 2019; Muñoz-Tobar y Catalino 2019). Los páramos también ofrecen servicios ecosistémicos como recolección, regulación y suministración de agua, muestra bellos paisajes para el ecoturismo, son esponjas naturales que sustentan de agua a grandes ciudades y retienen carbono orgánico (Ortiz y Mena, 2002; Buyataert et al., 2006; Castro, 2011). Estudios realizados sobre la importancia de la biodiversidad en los páramos (Cadena-Ortiz et al. 2019; García et. al 2019) demuestran que es necesario desarrollar estrategias para conservarlo. El presente estudio presenta el inventario de la diversidad de vertebrados terrestres en el ecosistema Herbazal húmedo subnival del páramo de la Reserva de Producción de Fauna Chimborazo (RPFCh), y analiza los índices de diversidad alfa y beta de la fauna registrada. Existen pocos estudios sobre la fauna presente en la Reserva (EcoCiencia, 2014), y otros se centran únicamente en poblaciones de Vicuñas, (McLaren y MacNearney 2013). A largo plazo, esperamos que los resultados de este estudio sean útiles como base para futuras investigaciones sobre ecología de comunidades de animales de páramo, monitoreo y conservación de las especies en este ecosistema. La identificación de especies permitirá conocer las localidades donde se encuentra la fauna silvestre y permite a las familias de comunidades aledañas generar ingresos con actividades ecoturísticas.

\section{Métodos.}

\section{Área de estudio.}

La Reserva de Producción de Fauna Chimborazo (RPFCh) fue creada en 1987 para la conservación y el manejo sustentable de la vida silvestre, la educación ambiental, la restauración de ecosistemas y el turismo de naturaleza, su denominación se debe al nevado "Chimborazo" con 6310 m.s.n.m de altitud (Arroyo y Cavieres, 2013). La RPFCh tiene una extensión de 58.560 has., abarca 38 organizaciones campesinas, está ubicada en las provincias de Tungurahua, Bolívar y Chimborazo, su temperatura varía entre $-0,11^{\circ} \mathrm{C}$ y $11,4^{\circ} \mathrm{C}$, sus precipitaciones van de $909 \mathrm{~mm}$ en verano y $1300 \mathrm{~mm}$ en invierno, diciembre 
es considerado el mes más seco, a diferencia de marzo y abril los más lluviosos (EcoCiencia, 2014). Según la clasificación del MAE, 2013 existen ocho ecosistemas: 1). Arbustal siempre verde y herbazal de páramo (3300-3900 m.s.n.m.), 2) Bosque siempre verde de páramo (3200-4100 m.s.n.m.), 3) Herbazal de páramo (3400-4300 ms.n.m.), 4) Herbazal húmedo montano alto superior de páramo (3500-4200 m.s.n.m.), 5) Herbazal húmedo subnival de páramo (3400-4300 m.s.n.m.), 6) Herbazal inundable de páramo (3300-4500 m. s.n.m.), 7) Herbazal y arbustal siempre verde subnival de páramo (44004900 m.s.n.m.), y 8) Herbazal ultrahúmedo subnival de páramo (4100-4500 m.s.n.m).

Figura 1. Mapa de localización del ecosistema Herbazal húmedo subnival del páramo perteneciente a la Reserva de Producción de Fauna Chimborazo.

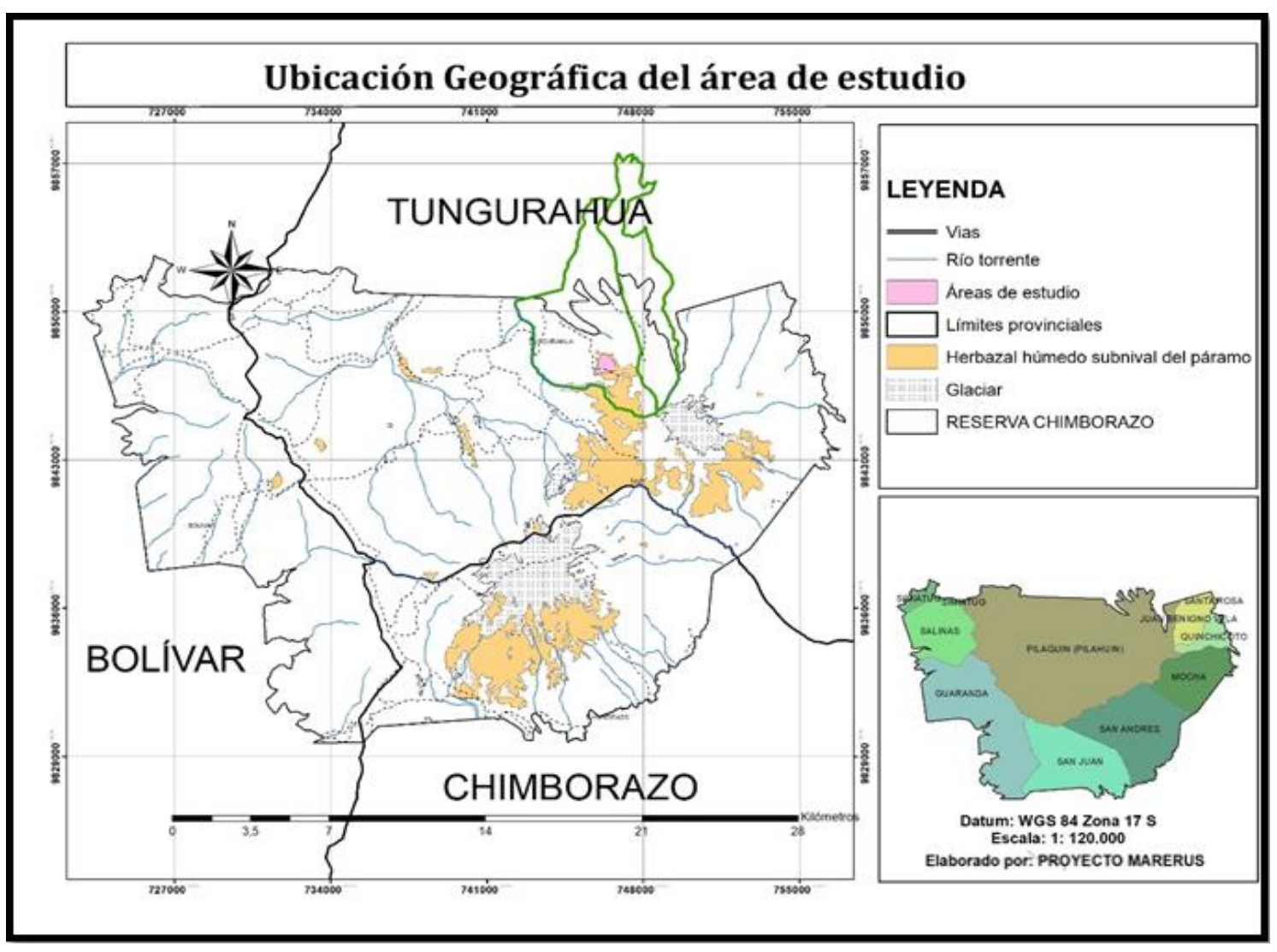

Fuente: Elaboración propia.

El estudio se desarrolló entre marzo y junio del 2019, identifica aves y mamíferos terrestres presentes en el ecosistema Herbazal húmedo subnival del páramo, emplea métodos y técnicas para la observación directa e indirecta (Gallina y López, 2011; Ministerio de Ambiente del Perú, 2015). Para identificar a los animales se utilizaron las guías de campo del Ecuador: mamíferos (Tirira, 2017), aves (Navarrete y McMullan 2018). Este trabajo está en el marco del proyecto "Medidas ante los riesgos que enfrentan los ecosistemas de la Reserva de Producción de Fauna Chimborazo frente al cambio de uso de suelo", que ejecuta la Facultad de Recursos Naturales (FRN), con financiamiento 
de la Escuela Superior Politécnica de Chimborazo (ESPOCH) con el fin de establecer nuevas estrategias de manejo sostenible para la conservación de la fauna silvestre. El ecosistema Herbazal húmedo subnival del páramo se localiza entre las coordenadas $\mathrm{X}$ : 746779 y Y: 9842679 . Tiene una temperatura entre los $6^{\circ} \mathrm{C}$ y $8^{\circ} \mathrm{C}$. La precipitación promedio anual es $998 \mathrm{~mm}$, en lugares más altos es en forma de nieve o escarcha. Este ecosistema tiene una altitud de 3400 a 4300 m.s.n.m. y una extensión de 4162,59 has. Según Sierra et al 1999, tiene una formación vegetal de páramo de almohadillas, y según Josse et al. 2003, tiene Vegetación geliturbata y edafoxerófila subnival paranuma (MAE, 2013).

Se definió dos sitios de muestreo, el primero está localizado en Pampas de Salasaca a una altitud media de $3950 \mathrm{msnm}$, su temperatura es de $6^{\circ} \mathrm{C}$, ubicado entre dos elevaciones en cuyas laderas se puede observar pequeños parches de bosque y una vegetación poco abundante debido a la presencia de ganado vacuno, esta zona de estudio es mayormente nublada y con presencia de lluvia. El segundo sitio se localiza en el sector Siete Cochas a una altitud media de $3860 \mathrm{msnm}$., su temperatura es de $8^{\circ} \mathrm{C}$, presenta una estructura más compacta con una vegetación más abundante, poca presencia de lluvia y ocasionalmente presencia de neblina. Se realizó parcelas de 300 x 300 m, éstas fueron seleccionadas para evitar efecto borde, sitios de difícil acceso, vías y poblados. Para mamíferos se aplicó transectos lineales que fueron observados de 7:30am hasta las 11:30am y por la tarde en horas de 15:00pm hasta 18:30pm, también se utilizó métodos indirectos como: seguimiento de rastro, huellas, restos alimenticios, heces, huesos y pelos, y adicionalmente, se instaló cámaras trampa en diferentes puntos estratégicos, utilizando cebos como: avena, atún, sardina, mantequilla de maní y esencia de vainilla. Para aves, se trazó transectos lineales de $1 \mathrm{~km}$ de distancia, se aplicó punto de conteo y conteo directo de 6:00 am a 10:30 am y de 16:00 a 18:30 pm; además, se utilizó recuentos en punto sin estimación de distancia y búsqueda intensiva, se tomó 20 minutos por cada punto de observación, y se complementó con caminatas al azar. Para los análisis estadísticos se utilizó el software informático Stimate 9.0; para la riqueza específica se realizó el índice de Margalef; para diversidad, se aplicó los índices de Shannon-Weaver, (Shannon y Weaver, 1948) y Simpson, éstos explican el patrón de disminución a gran escala de la riqueza de especies desde latitudes ecuatoriales bajas hasta latitudes templadas altas para la fauna terrestre (Simpson 1964; Ojeda 2013). La interpretación de los valores de diversidad de Simpson y Shannon se fundamentó en parámetros establecidos según Moreno 2001.

\section{Resultados.}

\section{Inventario de vertebrados terrestres.}

En el ecosistema Herbazal húmedo subnival del páramo se registraron un total de 17 especies, aves (6 familias, 4 órdenes y 10 especies); mamíferos (6 familias, 5 órdenes y 7 especies). 
Tabla 1. Vertebrados terrestres del ecosistema Herbazal húmedo subnival del páramo

\begin{tabular}{|c|c|c|c|c|}
\hline \multicolumn{5}{|c|}{ Mamíferos } \\
\hline No & Orden & Familia & Nombre científico & Nombre común \\
\hline 1 & Rodentia & Cricetidae & Akodon mollis & $\begin{array}{l}\text { Ratón campestre } \\
\text { delicado }\end{array}$ \\
\hline 2 & Lagomorpha & Leporidae & Sylvilagus andinus & Conejo Andino \\
\hline 3 & Carnivora & Canidae & Pseudalopex culpaeus & Zorro Andino \\
\hline 4 & Artiodactyla & Camelidae & Vicugna vicugna & Vicuña \\
\hline 5 & Artiodactyla & Camelidae & Lama glama & Llama \\
\hline 6 & Artiodactyla & Cervidae & Odocoileus ustus & Venado de cola blanca \\
\hline 7 & Eulipotyphla & Soricidae & Cryptotis montivagus & $\begin{array}{l}\text { Musaraña ecuatoriana } \\
\text { canosa }\end{array}$ \\
\hline \multicolumn{5}{|c|}{ Aves } \\
\hline 1 & Paseriformes & Furnariidae & Cinclodes excelsior & Chungui grande \\
\hline 2 & Paseriformes & Furnariidae & Asthenes wyatti & Canastero de Wyatt \\
\hline 3 & Paseriformes & Furnariidae & Asthenes flammulata & Canastero Flamulado \\
\hline 4 & Paseriformes & Turdidae & Turdus chiguanco & Mirlo Negro \\
\hline 5 & Passeriforme & Passerellidae & Zonotrichia capensis & Gorrión chingolo \\
\hline 6 & Falconiformes & Falconidae & $\begin{array}{l}\text { Phalcoboenus } \\
\text { carunculatus }\end{array}$ & Caracara curiquingue \\
\hline 7 & Paseriformes & Furnariidae & Cinclodes fuscus & Chunguichico \\
\hline 8 & Caprimulgiformes & Trochilidae & $\begin{array}{l}\text { Oreotrochilus } \\
\text { chimborazo } \\
\text { chimborazo }\end{array}$ & $\begin{array}{l}\text { Estrella del } \\
\text { Chimborazo }\end{array}$ \\
\hline 9 & Passeriformes & Thraupidae & Diglossa humeralis & Pinchaflor negro \\
\hline 10 & Apodiformes & Trochilidae & Lesbia victoriae & Colibrí colacinta \\
\hline
\end{tabular}

Fuente: Elaboración propia.

\section{Análisis de los índices de diversidad.}

\section{Mamíferos riqueza y abundancia.}

El índice de Margalef expresa la riqueza específica (número de especies) y fue de 1,484 indica una diversidad baja, se registró 7 especies, y una abundancia de 57 individuos. La abundancia relativa se expresa como la división entre el número de especies observadas de una misma comunidad y el total de especies observadas, en este caso se obtuvo para la especie Akodon mollis una abundancia relativa de " 0,05 " mientras que para las especies Sylvilagus andinus "0,14", Pseudalopex culpaeus " 0,05 ", Vicugna vicugna “0,42”, Lama glama “0,26”, Odocoileus ustus “0,03”, y Cryptotis montivagus "0,03”, dando como resultado 1. (Tabla 2). El índice de Simpson dio 0,726, diversidad baja, puesto que Vicugna vicugna es dominante $(0,42)$. El índice de equidad Shannon-Wiener fue 1,536, diversidad media baja, Vicugna vicugna al ser especie dominante hace que disminuya la equidad. 
Tabla 2. Cálculo de riqueza y abundancia relativa para mamíferos

\begin{tabular}{ccccccc}
\hline $\mathbf{N}^{\mathbf{0}}$ & Especies & Número individuos & $\begin{array}{c}\text { Abundancia } \\
\text { relativa (pi) }\end{array}$ & $\mathbf{p i}^{\wedge} \mathbf{2}$ & $\mathbf{l n}(\mathbf{p i})$ & $\begin{array}{c}\mathbf{p i} \\
(\mathbf{l n p i})\end{array}$ \\
\hline $\mathbf{1}$ & Akodon mollis & 3 & 0,0526 & 0,003 & $-2,944$ & $-0,155$ \\
$\mathbf{2}$ & Sylvilagus andinus & 8 & 0,1404 & 0,020 & $-1,964$ & $-0,276$ \\
$\mathbf{3}$ & Pseudalopex culpaeus & 3 & 0,0526 & 0,003 & $-2,944$ & $-0,155$ \\
$\mathbf{4}$ & Vicugna vicugna & 24 & 0,4211 & 0,177 & $-0,865$ & $-0,364$ \\
$\mathbf{5}$ & Lama glama & 15 & 0,2632 & 0,069 & $-1,335$ & $-0,351$ \\
$\mathbf{6}$ & Odocoileus ustus & 2 & 0,0351 & 0,001 & $-3,350$ & $-0,118$ \\
$\mathbf{7}$ & Cryptotis montivagus & 2 & 0,0351 & 0,001 & $-3,350$ & $-0,118$ \\
$\mathbf{S}$ & 7 & 57 & 1 & 0,274 & & $-1,536$ \\
I. Simpson & 0,726 & Diversidad baja & & & & \\
I. Shannon & 1,536 & Diversidad media baja & & & & \\
I. Margalef & 1,484 & Diversidad baja & & & & \\
\hline
\end{tabular}

S (Riqueza específica)

Fuente: Elaboración propia.

\section{Aves riqueza y abundancia.}

El índice de Margalef expresa la riqueza específica (número de especies) y fue de 2,364 indica una diversidad media baja, se registró 10 especies, y una abundancia de 45 individuos. La abundancia relativa de la especie Cinclodes excelsior fue de " 0,40 " mientras que para las especies Asthenes wyatti " 0,06 ", Asthenes flammulata"0,04", Turdus chiguanco " 0,13 ”, Zonotrichia capensis " 0,11 ”, Phalcoboenus carunculatus “0,02”, Cinclodes fuscus " 0,06 ", Oreotrochilus chimborazo chimborazo " 0,08 ", Diglossa humeralis " 0,02 ", Lesbia victoriae " 0,04 ", dando como resultado 1. (Tabla 2). El índice de Simpson dio 0,788, diversidad baja, puesto que "Cinclodes excelsior" $(0,40)$ es dominante. El índice de equidad Shannon-Wiener fue 1,901, diversidad media baja, "Cinclodes excelsior" al ser especie dominante hace que disminuya la equidad.

Tabla 3. Cálculo de riqueza y abundancia relativa para aves

\begin{tabular}{ccccccc}
\hline $\mathbf{N}^{\mathbf{0}}$ & Especies & $\begin{array}{c}\text { Número } \\
\text { individuos }\end{array}$ & $\begin{array}{c}\text { Abundancia } \\
\text { relativa (pi) }\end{array}$ & $\mathbf{p i}^{\wedge} \mathbf{2}$ & $\mathbf{l n}(\mathbf{p i})$ & pi (lnpi) \\
\hline $\mathbf{1}$ & Cinclodes excelsior & 18 & 0,400 & 0,160 & $-0,916$ & $-0,367$ \\
$\mathbf{2}$ & Asthenes wyatti & 3 & 0,067 & 0,004 & $-2,708$ & $-0,181$ \\
$\mathbf{3}$ & Asthenes flammulata & 2 & 0,044 & 0,002 & $-3,114$ & $-0,138$ \\
$\mathbf{4}$ & Turdus chiguanco & 6 & 0,133 & 0,018 & $-2,015$ & $-0,269$ \\
$\mathbf{5}$ & Zonotrichia capensis & 5 & 0,111 & 0,012 & $-2,197$ & $-0,244$ \\
$\mathbf{6}$ & Phalcoboenus & 1 & 0,022 & 0,000 & $-3,807$ & $-0,085$ \\
$\mathbf{7}$ & carunculatus & & & & & \\
& Cinclodes fuscus & 3 & 0,067 & 0,004 & $-2,708$ & $-0,181$ \\
\hline
\end{tabular}




\begin{tabular}{ccccccc}
\hline $\mathbf{8}$ & $\begin{array}{c}\text { Oreotrochilus } \\
\text { chimborazo } \\
\text { Chimborazo }\end{array}$ & 4 & 0,089 & 0,008 & $-2,420$ & $-0,215$ \\
$\mathbf{9}$ & $\begin{array}{c}\text { Diglossa humeralis } \\
\mathbf{1 0}\end{array}$ & 1 & 0,022 & 0,000 & $-3,807$ & $-0,085$ \\
Sesbia victoriae & 2 & 0,044 & 0,002 & $-3,114$ & $-0,138$ \\
I. Simpson & 10 & 45 & 1 & 0,212 & & $-1,901$ \\
I. Shannon & 0,788 & Diversidad baja & & & \\
I. Margalef & 1,901 & Diversidad media baja & & & \\
\hline
\end{tabular}

S (Riqueza específica)

Fuente: Elaboración propia.

\section{Discusión.}

Los datos muestran que a pesar de que el ecosistema Herbazal húmedo Subnival del páramo se ubica a una elevada altitud (3400-4300 m.s.n.m), siete especies de mamíferos fueron registrados. El Plan de Manejo actualizado de la RPFCH (2014) reportó en total 18 especies para toda la Reserva. La extensión del sitio de estudio representa sólo el 7\% del área total de la reserva, sin embargo, se encontró un tercio del total de las especies registradas en este ecosistema. Las aves registradas para la Reserva es 62 especies, este trabajo registró 10 especies. La diversidad media baja (Índice de Shannon) para mamíferos y aves, se debe a la presencia de especies dominantes como Vicugna vicugna y Cinclodes excelsior, añadido a las fluctuaciones de las condiciones climáticas como temperatura, precipitación y humedad. Diversos estudios (Patterson et al. 2003; Burneo 2011; Brito et al. 2017; Sklenár 2008; Camacho et al., 2019) señalan que mientras aumenta la altitud, la diversidad y riqueza tienden a disminuir; sin embargo, hay excepciones como el grupo taxonómico Rodentia de la familia Cricetidae que no muestran un patrón de diversidad decreciente en relación con aumento de elevación altitudinal (Burneo, 2011), esto podría deberse al tipo de alimentación de los Rodentia que en muchos casos es una dieta generalista (Brito y Ojala-Barbor, 2016), también su pequeño tamaño y su denso pelaje les permite habitar superficies con baja temperatura. Estudios en páramo muestran que la diversidad animal dependerá del tipo de formación vegetal como la de bosque, éstos guardan microclimas al interior y mantienen temperaturas constantes y son menos susceptibles a las heladas de un páramo Herbazal (Cierjacks et al. 2007). Otras investigaciones muestran que especies del género Thomasomys son generalistas de hábitat y dominan diferentes ecosistemas y áreas perturbadas mientras que hay especies especialistas de hábitat que son más vulnerables a pérdida de heterogeneidad y complejidad de hábitats verticales (Ojala-Barbour et al. 2019); la presencia de especies dentro de una matriz de hábitats depende de la capacidad de individuos para dispersarse, sobrevivir y reproducirse. 
El grupo Rodentia como los Lagomorpha son importantes para la dispersión de semillas en el páramo y al evidenciarlos en este trabajo, se recomienda realizar estudios sobre ecología de poblaciones debido que son especies claves que sirven como alimento de carnívoros y aves rapaces (Cadena-Ortiz et al. 2019), puesto que a elevadas alturas el número disponible de presas disminuye (García et al. 2016). Los herbazales se localizan en las partes más altas de las montañas de los Andes ecuatorianos, si comparamos la formación vegetal de los herbazales estos se componen por pastos de tallo corto, rosetas acaulescentes y hierbas de cojín (MAE, 2013), los cojines edafoxerófilos (Josse et al. 2003) es un grupo diverso adaptado fisiológicamente a las condiciones climáticas extremas (Körner 2003; Sklenár y Balslev 2007) lo que dificulta que especies de gran tamaño puedan mimetizarse en medio de la vegetación. Este trabajo registró 10 especies de aves, el Plan de Manejo de la Reserva registra 62, la dieta de las aves está asociada a su distribución, en este ecosistema se ubican especies de pequeño tamaño, siendo dominante la Cinclodes excelsior. Nos gustaría expresar nuestro agradecimiento a Eduardo Muñoz, director del proyecto "Medidas ante los riesgos que enfrentan los ecosistemas de la Reserva de Producción de Fauna Chimborazo frente al cambio de uso de suelo" (MARERUS) que ejecuta la Facultad de Recursos Naturales (FRN), con financiamiento de la Escuela Superior Politécnica de Chimborazo (ESPOCH) quienes nos proporcionaron los fondos y experiencia que contribuyeron en esta investigación, agradecer especialmente a David Gusñay por la información obtenida en las salidas de campo y a la Dirección de Escuela de Turismo de la FRN por proporcionar las cámaras trampas utilizadas en esta investigación.

\section{Conclusiones:}

- El ecosistema Herbazal húmedo subnival de páramo de la reserva de producción de fauna Chimborazo, es un ambiente natural en el cual se inventariaron 17 especies faunísticas, de las cuales el $41 \%$ son mamíferos y el $59 \%$ aves, registradas durante el proceso de investigación. Además, existe presencia de ganado vacuno y otros animales como perros que habitan en la reserva, constituyéndose una amenaza para la fauna silvestre.

- En el área de estudio, se inventariaron 7 especies de mamíferos: Akodon mollis, Sylvilagus andinus, Pseudalopex culpaeus, Vicugna vicugna, Lama glama, Odocoileus ustus, Cryptotis montivagus, y se obtuvo una abundancia total de 57 individuos de las mismas. Mediante el cálculo de los índices de diversidad se obtuvieron valores de: Simpson: 0,726, Shannon: 1,536 y Margalef; 1,484, lo que refleja una diversidad baja y una dominancia media alta. Y se inventario 10 especies de aves: Cinclodes excelsior, Asthenes wyatti, Asthenes flammulata, Turdus chiguanco, Zonotrichia capensis, Phalcoboenus carunculatus, Cinclodes fuscus, Oreotrochilus chimborazo Chimborazo, Diglossa humeralis, Lesbia victoriae, y se obtuvo una abundancia total de 45 individuos de las mismas. Mediante el cálculo de los índices de diversidad se obtuvieron valores de: 
Simpson: 0,788, Shannon: 1,901 y Margalef; 2,364, lo que refleja una diversidad media y una dominancia media alta.

- Las especies dominantes dentro del ecosistema de estudio, se ven marcadas por mamíferos medianos y grandes, siendo la especie Vicugna vicugna la más representativa, y para aves la especie más representativa es Cinclodes excelsior. En el caso de herpetofauna no se encontró ninguna especie en el área.

\section{Referencias bibliográficas:}

Arroyo, M. T. K., \& Cavieres, L. A. (2013). High-elevation Andean ecosystems.

Benítez, Á., Gradstein, S. R., Cevallos, P., Medina, J., \& Aguirre, N. (2019). Comunidades de briófitos terrestres relacionados con factores climáticos y topográficos en un páramo del sur de Ecuador. Caldasia, 41(2), 370-379. https://doi.org/10.15446/caldasia.v41n2.67869

Beltrán, K., Salgado, S., Cuesta, F., León-Yánez, S., Romoleroux, K., Ortiz, E., Cárdenas, A., \& Velástegui, A. (2009). Distribución espacial, sistemas ecológicos y caracterización florística de los páramos en el Ecuador. Quito: EcoCiencia, Proyecto Páramo Andino y Herbario QCA.

Bioweb (2020). https://bioweb.bio/faunaweb.html

Bravo, E. (2014). La biodiversidad en el Ecuador. Quito, Editorial ABYA YALA, Universidad Politécnica Salesiana.

Brito, J., Batallas, D., \& Yánez-Muñoz, M. H. (2017). Ranas terrestres Pristimantis (Anura: Craugastoridae) de los bosques montanos del río Upano, Ecuador: Lista anotada, patrones de diversidad y descripción de cuatro especies nuevas. Neotropical Biodiversity, 3(1), 125-156.

Brito, J., \& Ojala-Barbour, R. (2016). Mamíferos no voladores del Parque Nacional Sangay, Ecuador. Papéis Avulsos de Zoologia, 56(5), 45-61.

Burneo Núñez, S. F. (2011). Small Mammals of Sangay National Park, Chimborazo Province and Morona Santiago Province, Ecuador.

Buytaert, W., Célleri, R., De Bièvre, B., \& Cisneros, F. (2006). Hidrología del páramo andino: propiedades, importancia y vulnerabilidad. Cuenca. Recuperado: Http://Www. Paramo. Org/Files/Hidrologia_paramo. Pdf.

Cadena-Ortiz, H., Solórzano, M. F., Noboa, M., \& Brito, J. (2019). Diet of the ShortEared Owl (Asio flammeus) in the Antisana highlands, Ecuador. HUITZIL: Journal of Mexican Ornithology, 20(2).

Camacho, L. F., Chávez, P., \& Tirira, D. G. (2019). Elevation and wind exposure shape the habitat preferences of the Andean cottontail Sylvilagus andinus (Lagomorpha: Leporidae). Mammalian Biology, 94(1), 1-3.

Castro, M., \& Ejecutoras, I. (2011). Proyecto Creación de Capacidades para Valoración Socio-económica de los humedales altoandinos. La Experiencia En OñaSaraguro-Yacuambi y El Frente Sur Occidental de Tungurahua. 
Collot, J.-Y., Sallarès, V., \& Pazmiño, N. (2009). Geologia y geofisica marina y terrestre del Ecuador: desde la costa continental hasta las Islas Galapagos.

EcoCiencia. (2014). Actualización del Plan de Manejo de la Reserva de Producción de Fauna Chimborazo. Informe final de consultoría. Riobamba.

Farley, K. A., Kelly, E. F., \& Hofstede, R. G. M. (2004). Soil organic carbon and water retention after conversion of grasslands to pine plantations in the Ecuadorian Andes. Ecosystems, 7(7), 729-739.

Freile, J. F., Solano-Ugalde, A., Brinkhuizen, D. M., Greenfield, P. J., Lysinger, M., Nilsson, J., Navarrete, L., \& Ridgely, R. S. (2017). Rare birds in Ecuador: third report of the Committee for Ecuadorian Records in Ornithology (CERO). Revista Ecuatoriana de Ornitología, 1.

Gallina Tessaro, S., \& López González, C. (2011). Manual de técnicas para el estudio de la fauna. Universidad Autónoma de Querétaro e Instituto de Ecología, AC México. 390p.

García, V. J., Márquez, C. O., Isenhart, T. M., Rodríguez, M., Crespo, S. D., \& Cifuentes, A.

G. (2019). Evaluating the conservation state of the páramo ecosystem: An object-based image analysis and CART algorithm approach for central Ecuador. Heliyon, 5(10), e02701.

García, J., Suárez, E., \& Zapata-Ríos, G. (2016). An assessment of the populations of Sylvilagus brasiliensis andinus in Páramos with different vegetation structures in the northeastern Andes of Ecuador. Neotropical Biodiversity, 2(1), 72-80.

Hofstede, R., Coppus, R., Vásconez, P. M., Segarra, P., Wolf, J., \& Sevink, J. (2002). El estado de conservación de los páramos de pajonal en el Ecuador. Ecotropicos, 15(1), 3-18.

Josse, C., Navarro, G., Comer, P., Evans, R., Faber-Langendoen, D., Fellows, M., Kittel, G., Menard, S., Pyne, M., \& Reid, M. (2003). Ecological systems of Latin America and the Caribbean. A Working Classification of Terrestrial Systems. NatureServe.

Leon-Garcia, I. V, \& Lasso, E. (2019). High heat tolerance in plants from the Andean highlands: Implications for paramos in a warmer world. PloS One, 14(11).

Lozano, P. (2017). Valoración económica del carbono capturado en el suelo de los bofedales

de la Reserva de Producción de Fauna Chimborazo (Tesis de postgrado. Master en Medio Ambiente). Escuela Superior Politécnica de Chimborazo). Riobamba.

Luteyn, J. L., Churchill, S. P., Griffin, D., Gradstein, S. R., Sipman, H. J. M., \& A., M. R. G. (1999). Páramos: a checklist of plant diversity, geographical distribution, and botanical literature (Vol. 84). New York Botanical Garden Press Bronx, New York, USA.

Luteyn, J. L., \& Balslev, H. (1992). Páramo: an Andean ecosystem under human influence. Academic Press London, UK. 
McMullan, M., \& Navarrete, L. (2017). Fieldbook of the Birds of Ecuador including the Galápagos Islands and common mammals. Ratty Ediciones, Quito.

McLaren, B. E., MacNearney, D., \& Siavichay, C. A. (2018). Livestock and the functional habitat of vicuñas in Ecuador: a new puzzle. Ecosphere, 9(1), e02066.

Mena, P., \& Medina, G. (2001). La biodiversidad de los páramos en el Ecuador. Los Páramos Del Ecuador, Particularidades, Problemas y Perspectivas, 27-53.

Ministerio de Ambiente Ecuador. (2014). Ministerio de Ambiente del Ecuador. Sistema Nacional de Áreas Protegidas. DOCUMENTOS. Plan de Manejo.

Ministerio de Ambiente Ecuador. (2013). Sistema de clasificación de ecosistemas del Ecuador Continental.

Ministerio del Ambiente, Perú. (2015). Guía de inventario de la fauna silvestre.

Moreno, C. (2001). Métodos para medir la biodiversidad. M\&T-Manuales y Tesis SEA, vol. 1. Zaragoza, 84(922495). Recuperado el 25 de marzo de 2019. Disponible en:https://www.researchgate.net/profile/Claudia_Moreno7/publication/30434666 6_Metodos_para_medir_la_biodiversidad/links/576c346e08aedb18f3eb262a/Me todos-para-medir-la-biodiversidad.pdf

Morales-Castilla, I., \& García-Valdés, R. (2014). Gradientes latitudinales de diversidad inversos, ¿excepciones que prueban la regla? Revista Ecosistemas, 23(1), 4-12.

Morales-Castilla, I., \& García-Valdés, R. (2014). Gradientes latitudinales de diversidad inversos, $;$ excepciones que prueban la regla? Revista Ecosistemas, 23(1), 4-12.

Mora, C., Tittensor, D. P., Adl, S., Simpson, A. G. B., \& Worm, B. (2011). How many species are there on Earth and in the ocean? PLoS Biology, 9(8).

Muñoz-Tobar, S. I., \& Caterino, M. S. (2019). The role of dispersal for shaping phylogeographical structure of flightless beetles from the Andes. PeerJ, 7, e7226.

Ojala-Barbour, R., Brito, J., \& Teska, W. R. (2019). A comparison of small mammal communities in two High-Andean Polylepis woodlands in Ecuador. ACI Avances En Ciencias e Ingenierías, 11(2).

Ojeda, R. A. (2013). Diversity and conservation of Neotropical mammals.

Ortega-Andrade, H. M., Prieto-Torres, D. A., Gomez-Lora, I., \& Lizcano, D. J. (2015). Ecological and geographical analysis of the distribution of the mountain tapir (Tapirus pinchaque) in Ecuador: importance of protected areas in future scenarios of global warming. PloS One, 10(3).

Ortiz, D., \& Vásconez, P. M. (2002). Las áreas protegidas y los páramos. GTP.

Schipper, J., Chanson, J. S., Chiozza, F., Cox, N. A., Hoffmann, M., Katariya, V., Lamoreux, J., Rodrigues, A. S. L., Stuart, S. N., \& Temple, H. J. (2008). The status of the world's land and marine mammals: diversity, threat, and knowledge. Science, 322(5899), 225-230. 
Shannon, C. E., \& Weaver, W. (1948). Teoría de la comunicación. Editorial Universidad Arturo Prat Sede Victoria. Chile.

Simpson, G. G. (1964). Species density of North American recent mammals. Systematic Zoology, 13(2), 57-73.

Tirira, D. (2017). Guía de Campo de los Mamíferos del Ecuador. Ediciones Murciélago Blanco. Publicación especial sobre los mamíferos del Ecuador (2nd ed.). QuitoEcuador: Murcielago Blanco.

Traveset, A., \& Santamaría, L. (2004). Alteración de mutualismos planta-animal debido a la introducción de especies exóticas en ecosistemas insulares. Ecología Insular, $251,276$. 


\section{PARA CITAR EL ARTÍCULO INDEXADO.}

Bayancela, S., \& Cajas, C. (2020). Vertebrados terrestres del ecosistema Herbazal húmedo subnival del páramo en la Reserva de Producción de Fauna Chimborazo. ConcienciaDigital, 3(3), 127-140. https://doi.org/10.33262/concienciadigital.v3i3.1285

\section{Liencia}

El artículo que se publica es de exclusiva responsabilidad de los autores y no necesariamente reflejan el pensamiento de la Revista Conciencia Digital.

El artículo queda en propiedad de la revista y, por tanto, su publicación parcial y/o total en otro medio tiene que ser autorizado por el director de la Revista Conciencia Digital.
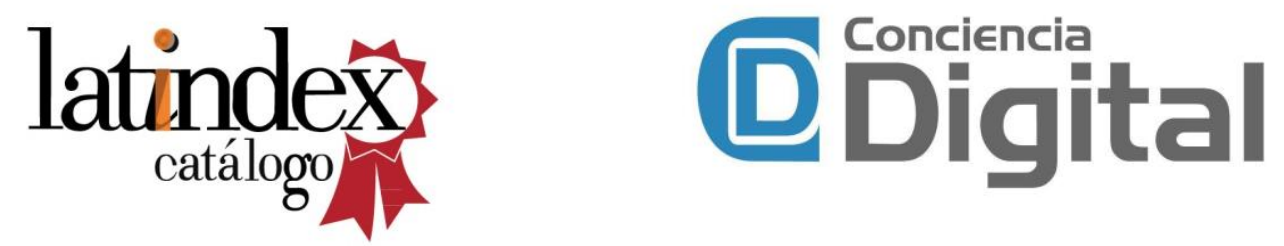